\title{
Existence of Solutions for a Class of Coupled Fractional Differential Systems with Nonlocal Boundary Conditions
}

\author{
Tingting Qi, ${ }^{1}$ Yansheng Liu, ${ }^{1}$ and Yujun $\mathrm{Cui}^{2}$ \\ ${ }^{1}$ School of Mathematics and Statistics, Shandong Normal University, Jinan, Shandong 250014, China \\ ${ }^{2}$ Department of Mathematics, Shandong University of Science and Technology, Qingdao, Shandong 266590, China \\ Correspondence should be addressed to Yansheng Liu; yanshliu@gmail.com
}

Received 6 April 2017; Accepted 5 June 2017; Published 6 July 2017

Academic Editor: Xinguang Zhang

Copyright ( 2017 Tingting Qi et al. This is an open access article distributed under the Creative Commons Attribution License, which permits unrestricted use, distribution, and reproduction in any medium, provided the original work is properly cited.

Applying Schauder fixed point theorem and Leray-Schauder nonlinear alternative theory, this paper is concerned with the existence of solutions to coupled fractional differential systems with fractional integral boundary value conditions. Meanwhile, two examples are worked out to illustrate the application of the main results.

\section{Introduction}

Fractional differential equations have a wide range of applications in many science and engineering, such as in physics, chemistry, biology, and electrodynamics. We refer the reader to see [1-5]. The main reason is that fractional derivatives provide an excellent tool for the description of memory and hereditary properties of various materials and processes. Therefore, this topic has attracted much attention of scientists and engineers. More and more good results are obtained. See [6-10] and references therein.

In recent years, fractional differential equations with the nonlinear terms involving fractional derivative $D_{0^{+}}^{\alpha}$ of unknown functions have been investigated by some authors. See [11-16] and references therein. For example, in [13], Su studied the following nonlinear coupled fractional differential systems:

$$
\begin{gathered}
D_{0^{+}}^{\alpha} u(t)=f\left(t, v(t), D_{0^{+}}^{\mu} v(t)\right), \quad t \in(0,1), \\
D_{0^{+}}^{\beta} v(t)=g\left(t, u(t), D_{0^{+}}^{\delta} u(t)\right), \quad t \in(0,1), \\
u(0)=u(1)=v(0)=v(1)=0,
\end{gathered}
$$

where $1<\alpha, \beta<2, \mu, \delta>0, \alpha-\delta \geq 1, \beta-\mu \geq$ 1 , and $f, g:[0,1] \times R \times R \rightarrow R$ are given continuous functions. $D_{0^{+}}^{\rho}(\rho=\alpha, \beta, \delta, \mu)$ is the standard RiemannLiouville fractional derivative. Applying Schauder fixed point theorem, the existence of solution was studied.

On the other hand, integral boundary conditions have various applications in applied fields such as chemical engineering, underground water flow, blood flow problems, thermoelasticity, population dynamics, and finite element method approaches with the minimization of constitutive error. In consequence, the integral boundary value problem of fractional differential equations is gaining much importance and attention. See [17-21] and references therein. For instance, in [19], Zhao and Liu studied the following coupled fractional differential systems with integral boundary conditions:

$$
\begin{aligned}
{ }^{C} D_{0^{+}}^{\alpha} u(t)+f(t, v(t)) & =0, \quad t \in(0,1), \\
{ }^{C} D_{0^{+}}^{\alpha} v(t)+g(t, u(t)) & =0, \quad t \in(0,1), \\
u^{(j)}(0) & =v^{(j)}(0)=0, \\
& 0 \leq j \leq n-1, \quad j \neq 1, \\
u^{\prime}(1) & =\lambda \int_{0}^{1} u(t) d t, \\
v^{\prime}(1) & =\lambda \int_{0}^{1} v(t) d t,
\end{aligned}
$$


where $n-1<\alpha \leq n, n \geq 3,0 \leq \lambda<2 .{ }^{C} D_{0^{+}}^{\alpha}$ is the Caputo fractional derivate and $f, g:[0,1] \times[0,+\infty) \rightarrow[0,+\infty)$ are given continuous functions. By using the monotone method and the theory of fixed point index on cone, they investigated the existence and uniqueness of solution for this coupled system.

In [21], Ahmad et al. investigated the following coupled fractional differential system with nonlocal and integral boundary value conditions:

$$
\begin{aligned}
{ }^{C} D_{0^{+}}^{\alpha} x(t) & =f\left(t, x(t), y(t),{ }^{C} D_{0^{+}}^{\gamma} y(t)\right), \\
{ }^{C} D_{0^{+}}^{\beta} y(t) & =g\left(t, x(t),{ }^{C} D_{0^{+}}^{\delta} x(t), y(t)\right), \\
t \in[0, T], & \\
t \in[0, T], & \\
\int_{0}^{T} y(0) & =h(y) d s=\mu_{1} x(\eta), \\
y(0) & =\phi(x), \\
\int_{0}^{T} x(s) d s & =\mu_{2} y(\xi),
\end{aligned}
$$

where $1<\alpha, \beta \leq 2,0<\gamma \leq \delta<1, \eta, \xi \in(0, T) . f, g$ : $[0, T] \times R \times R \times R \rightarrow R, h, \phi: C([0, T], R) \rightarrow R$ are given continuous functions and $\mu_{1}, \mu_{2}$ are real number. Applying Banach contraction mapping principle and Leray-Schauder nonlinear alternative theory, the existence and uniqueness of solution were studied.

To the best of our knowledge, there are fewer results for coupled fractional differential systems with nonlocal and fractional integral boundary value conditions. Motivated by the above-mentioned references, we consider the existence of solutions of the following systems:

$$
\begin{aligned}
{ }^{C} D_{0^{+}}^{\alpha} x(t) & =f\left(t, y(t),{ }^{C} D_{0^{+}}^{\gamma} y(t)\right), \quad t \in[0,1], \\
{ }^{C} D_{0^{+}}^{\beta} y(t) & =g\left(t, x(t),{ }^{C} D_{0^{+}}^{\delta} x(t)\right), \quad t \in[0,1], \\
x(0) & =h(x), \\
x(1) & =a I_{0^{+}}^{\theta_{1}} x(\eta), \\
y(0) & =\varphi(y), \\
y(1) & =b I_{0^{+}}^{\theta_{2}} y(\xi),
\end{aligned}
$$

where $I_{0^{+}}^{\theta_{i}}(i=1,2)$ is Riemann-Liouville fractional integral, $1<\alpha, \beta<2,0<\gamma, \delta<1,0 \leq \xi, \eta \leq 1, \theta_{i}>0(i=$ $1,2), a, b \in R . f, g \in C([0,1] \times R \times R \rightarrow R)$, and $h, \varphi:$ $C([0,1], R) \rightarrow R$ are given continuous functionals. Applying Schauder fixed point theorem and Leray-Schauder nonlinear alternative theory, some existence results of solutions to this systems are obtained. Finally, two examples are worked out to illustrate the application of our results.
The main features of this paper are as follows. (1) The coupled fractional differential systems with nonlocal and fractional integral boundary value conditions are first studied. (2) The nonlinear term here involves fractional derivative of unknown functions. (3) Nonlocal conditions such as $x(0)=$ $h(x)$ and $y(0)=\varphi(y)$ and fractional integral boundary conditions such as $x(1)=a I_{0^{+}}^{\theta_{1}} x(\eta)$ and $y(1)=b I_{0^{+}}^{\theta_{2}} y(\xi)$ are more extensive and superior to local conditions.

The rest of this paper is organized as follows. Section 2 introduces some basic definitions and lemmas. In Section 3, the main results are presented. Finally, in Section 4, some examples are given to illustrate the effectiveness of the main results.

\section{Preliminary Results}

In this section, we first introduce some definitions and lemmas for fractional calculus. For details, please refer to $[1,22]$.

Definition 1. The Caputo fractional derivative of order $\alpha>0$ of a function $u:(0, \infty) \rightarrow R$ is given by

$$
{ }^{C} D_{0^{+}}^{\alpha} u(t)=\frac{1}{\Gamma(n-\alpha)} \int_{0}^{t} \frac{u^{(n)}(s)}{(t-s)^{\alpha-n+1}} d s,
$$

where $n=[\alpha]+1,[\alpha]$ denotes the integer part of the real number $\alpha$.

Definition 2. The Riemann-Liouville fractional integral of order $\alpha>0$ of a function $u:(0, \infty) \rightarrow R$ is given by

$$
I_{0^{+}}^{\alpha} u(t)=\frac{1}{\Gamma(\alpha)} \int_{0}^{t}(t-s)^{\alpha-1} u(s) d s,
$$

where $\Gamma$ is the gamma function.

Lemma 3. Let $\alpha>0$. Then the fractional differential equation ${ }^{C} D_{0^{+}}^{\alpha} u(t)=0$ has solution

$$
u(t)=c_{1}+c_{2} t+c_{3} t^{2}+\cdots+c_{n} t^{n-1},
$$

where $c_{i} \in R, i=1,2, \ldots, n, n=[\alpha]+1$.

Lemma 4. Let $n-1<\alpha \leq n(n \in N)$. Then

$$
I_{0^{+}}^{\alpha}{ }^{C} D_{0^{+}}^{\alpha} u(t)=u(t)+c_{1}+c_{2} t+c_{3} t^{2}+\cdots+c_{n} t^{n-1},
$$

where $c_{i} \in R, i=1,2, \ldots, n, n=[\alpha]+1$.

Lemma 5. Let $p>q \geq 0$, and $g \in L[a, b]$. Then

$$
\begin{aligned}
I_{0^{+}}^{p} I_{0_{+}}^{q} g(t) & =I_{0^{+}}^{p+q} g(t)=I_{0^{+}}^{q} I_{0^{+}}^{p} g(t), \\
{ }^{C} D_{0^{+}}^{p} I_{0^{+}}^{p} g(t) & =g(t), \\
{ }^{C} D_{0^{+}}^{q} I_{0^{+}}^{p} g(t) & =I_{0^{+}}^{p-q} g(t),
\end{aligned}
$$


Lemma 6. Suppose $h, \varphi: C[0,1] \rightarrow R$ are given continuous functionals and $\omega, u \in L[0,1]$. Then the boundary value problem

$$
\begin{aligned}
{ }^{C} D_{0^{+}}^{\alpha} x(t) & =\omega(t), \quad t \in[0,1], \\
{ }^{C} D_{0^{+}}^{\beta} y(t) & =u(t), \quad t \in[0,1], \\
x(0) & =h(x), \\
x(1) & =a I_{0^{+}}^{\theta_{1}} x(\eta), \\
y(0) & =\varphi(y), \\
y(1) & =b I_{0^{+}}^{\theta_{2}} y(\xi)
\end{aligned}
$$

is equivalent to system

$$
\begin{aligned}
x(t)= & \left(1+\tau_{1} t\right) h(x)+\frac{a t}{1-a \triangle_{1}} I_{0^{+}}^{\alpha+\theta_{1}} \omega(\eta) \\
& -\frac{t}{1-a \triangle_{1}} I_{0^{+}}^{\alpha} \omega(1)+I_{0^{+}}^{\alpha} \omega(t), \\
y(t)= & \left(1+\tau_{2} t\right) \varphi(y)+\frac{b t}{1-b \triangle_{2}} I_{0^{+}}^{\beta+\theta_{2}} u(\xi) \\
& -\frac{t}{1-b \triangle_{2}} I_{0^{+}}^{\beta} u(1)+I_{0^{+}}^{\beta} u(t),
\end{aligned}
$$

where $1<\alpha, \beta<2,0 \leq \xi, \eta \leq 1, \theta_{i}>0(i=1,2), a, b \in$ $R, 1-a \triangle_{1} \neq 0,1-b \triangle_{2} \neq 0$,

$$
\begin{aligned}
& \triangle_{1}=\frac{\eta^{\theta_{1}+1}}{\Gamma\left(\theta_{1}+2\right)}, \\
& \sigma_{1}=\frac{\eta^{\theta_{1}}}{\Gamma\left(\theta_{1}+1\right)}, \\
& \tau_{1}=\frac{a \sigma_{1}-1}{1-a \triangle_{1}}, \\
& \triangle_{2}=\frac{\xi^{\theta_{2}+1}}{\Gamma\left(\theta_{2}+2\right)}, \\
& \sigma_{2}=\frac{\xi^{\theta_{2}}}{\Gamma\left(\theta_{2}+1\right)}, \\
& \tau_{2}=\frac{b \sigma_{2}-1}{1-b \triangle_{2}} .
\end{aligned}
$$

Proof. In view of Lemmas 3 and 4 , the solution of ${ }^{C} D_{0^{+}}^{\alpha} x(t)=$ $\omega(t)$ is

$$
x(t)=c_{1}+c_{2} t+I_{0^{+}}^{\alpha} \omega(t),
$$

where $c_{i} \in R, i=1$, 2. From $x(0)=h(x)$, one can get $c_{1}=$ $h(x)$. By means of Lemma 5 and (14), we have

$$
I_{0^{+}}^{\theta_{1}} x(t)=\frac{c_{1} t^{\theta_{1}}}{\Gamma\left(\theta_{1}+1\right)}+\frac{c_{2} t^{\theta_{1}+1}}{\Gamma\left(\theta_{1}+2\right)}+I_{0^{+}}^{\alpha+\theta_{1}} \omega(t) .
$$

Since $x(1)=a I_{0^{+}}^{\theta_{1}} x(\eta)$, one has

$$
\begin{aligned}
c_{1}+c_{2}+I_{0^{+}}^{\alpha} \omega(1)= & \frac{a c_{1}}{\Gamma\left(\theta_{1}+1\right)} \eta^{\theta_{1}}+\frac{a c_{2}}{\Gamma\left(\theta_{1}+2\right)} \eta^{\theta_{1}+1} \\
& +a I_{0^{+}}^{\alpha+\theta_{1}} \omega(\eta),
\end{aligned}
$$

which implies

$$
\begin{aligned}
c_{2}\left(1-a \triangle_{1}\right)= & \left(a \sigma_{1}-1\right) h(x)+a I_{0^{+}}^{\alpha+\theta_{1}} \omega(\eta) \\
& -I_{0^{+}}^{\alpha} \omega(1) .
\end{aligned}
$$

Thus,

$$
\begin{aligned}
c_{1}= & h(x), \\
c_{2}= & \frac{a \sigma_{1}-1}{1-a \triangle_{1}} h(x)+\frac{a}{1-a \triangle_{1}} I_{0^{+}}^{\alpha+\theta_{1}} \omega(\eta) \\
& -\frac{1}{1-a \triangle_{1}} I_{0^{+}}^{\alpha} \omega(1) .
\end{aligned}
$$

Substituting $c_{1}$ and $c_{2}$ to (14), we obtain that (11) holds. Similarly, one can prove that (12) holds.

Let $X=\left\{x \in C[0,1]:{ }^{C} D_{0^{+}}^{\delta} x(t) \in C[0,1]\right\}$ be endowed with the norm $\|x\|_{X}=\|x\|_{c}+\left\|{ }^{C} D_{0^{+}}^{\delta} x\right\|_{\mathcal{c}}=\max _{t \in[0,1]}|x(t)|+$ $\left.\max _{t \in[0,1]}\right|^{C} D_{0^{+}}^{\delta} x(t) \mid$. Then we have the following conclusions.

Lemma 7. $\left(X,\|\cdot\|_{X}\right)$ is a Banach space

Let $Y=\left\{y \in C[0,1]:{ }^{C} D_{0^{+}}^{\gamma} y(t) \in C[0,1]\right\}$ be endowed with $\|y\|_{Y}=\|y\|_{\mathcal{c}}+\left\|{ }^{C} D_{0^{+}}^{\gamma} y\right\|_{\mathcal{c}}=\max _{t \in[0,1]}|y(t)|+$ $\max _{t \in[0,1]}\left|{ }^{C} D_{0^{+}}^{\gamma} y(t)\right|$. Then $\left(Y,\|\cdot\|_{Y}\right)$ is a Banach space. Let $\|(x, y)\|_{X \times Y}=\|x\|_{X}+\|y\|_{Y}$. Clearly $\left(X \times Y,\|\cdot\|_{X \times Y}\right)$ is also a Banach space.

Defining an operator $T: X \times Y \rightarrow X \times Y$ by

$$
\begin{array}{r}
T(x, y)(t)=\left(T_{1}(x, y)(t), T_{2}(x, y)(t)\right), \\
\forall x \in X, \quad y \in Y,
\end{array}
$$

where

$$
\begin{aligned}
T_{1}(x, y)(t) \\
=\left(1+\tau_{1} t\right) h(x) \\
\quad+\left.\frac{a t}{1-a \triangle_{1}} I_{0^{+}}^{\alpha+\theta_{1}} f\left(t, y(t),{ }^{C} D_{0^{+}}^{\gamma} y(t)\right)\right|_{t=\eta} \\
\quad-\left.\frac{t}{1-a \triangle_{1}} I_{0^{+}}^{\alpha} f\left(t, y(t),{ }^{C} D_{0^{+}}^{\gamma} y(t)\right)\right|_{t=1} \\
\quad+I_{0^{+}}^{\alpha} f\left(t, y(t),{ }^{C} D_{0^{+}}^{\gamma} y(t)\right), \\
T_{2}(x, y)(t) \\
=\left(1+\tau_{2} t\right) \varphi(y) \\
\quad+\left.\frac{b t}{1-b \triangle_{2}} I_{0^{+}}^{\beta+\theta_{2}} g\left(t, x(t),{ }^{C} D_{0^{+}}^{\delta} x(t)\right)\right|_{t=\xi}
\end{aligned}
$$




$$
\begin{aligned}
& -\left.\frac{t}{1-b \triangle_{2}} I_{0^{+}}^{\beta} g\left(t, x(t),{ }^{C} D_{0^{+}}^{\delta} x(t)\right)\right|_{t=1} \\
& +I_{0^{+}}^{\beta} g\left(t, x(t),{ }^{C} D_{0^{+}}^{\delta} x(t)\right),
\end{aligned}
$$

$\triangle_{i}, \sigma_{i}, \tau_{i}(i=1,2)$ are described as in Lemma 6.

Lemma 8. Suppose $f, g \in C\left([0,1] \times R^{2}, R\right)$. Then $(x, y) \in$ $X \times Y$ is a solution of problem (4) if and only if $(x, y) \in X \times Y$ is a fixed point of the operator $T$.

Proof. By Lemma 6, the necessity is obvious. Now we show sufficiency.

Suppose $(x, y) \in X \times Y$ is a fixed point of the operator $T$. This together with (19) indicates

$$
\begin{aligned}
x(t)= & \left(1+\tau_{1} t\right) h(x) \\
& +\left.\frac{a t}{1-a \triangle_{1}} I_{0^{+}}^{\alpha+\theta_{1}} f\left(t, y(t),{ }^{C} D_{0^{+}}^{\gamma} y(t)\right)\right|_{t=\eta} \\
& -\left.\frac{t}{1-a \triangle_{1}} I_{0^{+}}^{\alpha} f\left(t, y(t),{ }^{C} D_{0^{+}}^{\gamma} y(t)\right)\right|_{t=1} \\
& +I_{0^{+}}^{\alpha} f\left(t, y(t),{ }^{C} D_{0^{+}}^{\gamma} y(t)\right), \\
y(t)= & \left(1+\tau_{2} t\right) \varphi(y) \\
& +\left.\frac{b t}{1-b \triangle_{2}} I_{0^{+}}^{\beta+\theta_{2}} g\left(t, x(t),{ }^{C} D_{0^{+}}^{\delta} x(t)\right)\right|_{t=\xi} \\
& -\left.\frac{t}{1-b \triangle_{2}} I_{0^{+}}^{\beta} g\left(t, x(t),{ }^{C} D_{0^{+}}^{\delta} x(t)\right)\right|_{t=1} \\
& +I_{0^{+}}^{\beta} g\left(t, x(t),{ }^{C} D_{0^{+}}^{\delta} x(t)\right) .
\end{aligned}
$$

Notice that ${ }^{C} D_{0^{+}}^{\rho} t^{\rho-m}=0, m=1,2, \ldots, N$, where $N$ is the smallest integer greater than or equal to $\rho$. Therefore,

$$
\begin{aligned}
{ }^{C} D_{0^{+}}^{\alpha} x(t) & ={ }^{C} D_{0^{+}}^{\alpha}\left[I_{0^{+}}^{\alpha} f\left(t, y(t),{ }^{C} D_{0^{+}}^{\gamma} y(t)\right)\right] \\
& =f\left(t, y(t),{ }^{C} D_{0^{+}}^{\gamma} y(t)\right) .
\end{aligned}
$$

Similarly, one has

$$
\begin{aligned}
{ }^{C} D_{0^{+}}^{\beta} y(t) & ={ }^{C} D_{0^{+}}^{\beta}\left[I_{0^{+}}^{\beta} g\left(t, x(t),{ }^{C} D_{0^{+}}^{\delta} x(t)\right)\right] \\
& =g\left(t, x(t),{ }^{C} D_{0^{+}}^{\delta} x(t)\right) .
\end{aligned}
$$

By direct computation, we can easily get $x(0)=h(x), y(0)=$ $\varphi(y), x(1)=a I_{0^{+}}^{\theta_{1}} x(\eta), y(1)=b I_{0^{+}}^{\theta_{2}} y(\xi)$. Therefore, $(x, y) \epsilon$ $X \times Y$ is a solution of (4).

Lemma 9 ([23] (Leray-Schauder nonlinear alternative)). Let $F$ be a Banach space, $\Omega$ be a bounded open subset of $F$, and $0 \in \Omega, T: \bar{\Omega} \rightarrow F$ be a completely continuous operator. Then, either there exists $x \in \partial \Omega, \lambda>1$ such that $T x=\lambda x$ or there exists a fixed point $x^{*} \in \bar{\Omega}$.

\section{Main Results}

For convenience, some notations and assumptions are stated as follows:

$$
\begin{aligned}
A_{1}= & \left|\frac{a}{1-a \triangle_{1}}\right| \frac{1}{\Gamma\left(\theta_{1}+\alpha+1\right)}+\left|\frac{1}{1-a \triangle_{1}}\right| \frac{1}{\Gamma(\alpha+1)} \\
& +\frac{1}{\Gamma(\alpha+1)}, \\
A_{2}= & \left|\frac{b}{1-b \triangle_{2}}\right| \frac{1}{\Gamma\left(\theta_{2}+\beta+1\right)}+\left|\frac{1}{1-b \triangle_{2}}\right| \frac{1}{\Gamma(\beta+1)} \\
& +\frac{1}{\Gamma(\beta+1)},
\end{aligned}
$$$$
B_{1}=\left|1+\tau_{1}\right|+\frac{\left|\tau_{1}\right|}{\Gamma(2-\delta)},
$$$$
B_{2}=\left|1+\tau_{2}\right|+\frac{\left|\tau_{2}\right|}{\Gamma(2-\gamma)} \text {, }
$$$$
C_{1}=\left|\frac{a}{1-a \triangle_{1}}\right| \frac{1}{\Gamma\left(\theta_{1}+\alpha+1\right)}+\left|\frac{1}{1-a \triangle_{1}}\right| \frac{1}{\Gamma(\alpha+1)}
$$$$
+\frac{1}{\Gamma(\alpha)}
$$$$
C_{2}=\left|\frac{b}{1-b \triangle_{2}}\right| \frac{1}{\Gamma\left(\theta_{2}+\beta+1\right)}+\left|\frac{1}{1-b \triangle_{2}}\right| \frac{1}{\Gamma(\beta+1)}
$$$$
+\frac{1}{\Gamma(\beta)} \text {, }
$$$$
D_{1}=A_{1}+\frac{C_{1}}{\Gamma(2-\delta)}
$$$$
D_{2}=A_{2}+\frac{C_{2}}{\Gamma(2-\gamma)} \text {, }
$$$$
P_{1}=a_{1}+b_{1} \phi_{1}(Q)+c_{1} \psi_{1}(Q),
$$$$
P_{2}=a_{2}+b_{2} \phi_{2}(Q)+c_{2} \psi_{2}(Q) \text {. }
$$

$\left(H_{1}\right) f, g:[0,1] \times R^{2} \rightarrow R$ are continuous functions and there exist constants $k_{i}>0, l_{i}>0$, and $m_{i}, n_{i} \in$ $(0,1), i=1,2$, such that

$$
\begin{aligned}
&|f(t, u, v)| \leq k_{0}+k_{1}|u|^{m_{1}}+k_{2}|v|^{m_{2}}, \\
& \forall t \in[0,1], u, v \in R ; \\
&|g(t, u, v)| \leq l_{0}+l_{1}|u|^{n_{1}}+l_{2}|v|^{n_{2}}, \\
& \forall t \in[0,1], u, v \in R .
\end{aligned}
$$

$\left(H_{2}\right) h, \varphi: C[0,1] \rightarrow R$ are continuous functionals, $h(0)=$ $\varphi(0)=0$, and there exist constants $K, L>0, \mu, \lambda \in$ $(0,1)$ such that, for all $x, y \in C[0,1]$,

$$
\begin{aligned}
& |h(x)| \leq K\|x\|_{c}^{\mu}, \\
& |\varphi(y)| \leq L\|y\|_{c}^{\lambda} .
\end{aligned}
$$


$\left(H_{3}\right) f, g:[0,1] \times R^{2} \rightarrow R$ are continuous functions, and there exist $a_{i}, b_{i}, c_{i}, d_{i} \geq 0(i=1,2)$ and nondecreasing functions $\phi_{i}, \psi_{i} \in C([0,+\infty),[0,+\infty))(i=1,2)$, such that

$$
|f(t, u, v)| \leq a_{1}+b_{1} \phi_{1}(|u|)+c_{1} \psi_{1}(|v|),
$$

$\forall t \in[0,1], u, v \in R$

$|g(t, u, v)| \leq a_{2}+b_{2} \phi_{2}(|u|)+c_{2} \psi_{2}(|v|)$,

$\forall t \in[0,1], u, v \in R$.

$\left(H_{4}\right)$ There exists $Q>0$ such that $B_{1} K Q^{\mu}+D_{1} P_{1}+B_{2} L Q^{\lambda}+$ $D_{2} P_{2}<Q$, where $K, L, \mu, \lambda$ are described as in $\left(H_{2}\right)$.

Theorem 10. Assume that $\left(H_{1}\right)-\left(H_{2}\right)$ hold. Then FDE (4) has at least one solution.

Proof. Let

$$
B_{R}=\left\{(x, y) \in X \times Y \mid\|(x, y)\|_{X \times Y} \leq R\right\},
$$

where

$$
\begin{aligned}
R \geq & \max \left\{\left(8 B_{1} K\right)^{1 /(1-\mu)},\left(8 B_{2} L\right)^{1 /(1-\lambda)}, 8 k_{0} D_{1}, 8 l_{0} D_{2},\right. \\
& \left(8 k_{1} D_{1}\right)^{1 /\left(1-m_{1}\right)},\left(8 l_{1} D_{2}\right)^{1 /\left(1-n_{1}\right)},\left(8 k_{2} D_{1}\right)^{1 /\left(1-m_{2}\right)} \\
& \left.\left(8 l_{2} D_{2}\right)^{1 /\left(1-n_{2}\right)}\right\} .
\end{aligned}
$$

Now we prove that $T: B_{R} \rightarrow B_{R}$. In fact, for any $(x, y) \in$ $B_{R}$, it follows from Definition 2 and $\left(H_{1}\right)$ that

$$
\begin{aligned}
& \left|T_{1}(x, y)(t)\right| \leq\left|1+\tau_{1}\right||h(x)| \\
& +\left|\frac{a}{1-a \triangle_{1}} I_{0^{+}}^{\alpha+\theta_{1}} f\left(t, y(t),{ }^{C} D_{0^{+}}^{\gamma} y(t)\right)\right|_{t=\eta} \mid \\
& +\left|\frac{1}{1-a \triangle_{1}} I_{0^{+}}^{\alpha} f\left(t, y(t),{ }^{C} D_{0^{+}}^{\gamma} y(t)\right)\right|_{t=1} \mid \\
& +\left|I_{0^{+}}^{\alpha} f\left(t, y(t),{ }^{C} D_{0^{+}}^{\gamma} y(t)\right)\right| \leq\left|1+\tau_{1}\right| K R^{\mu} \\
& +\left|\frac{a}{1-a \triangle_{1}}\right| \frac{1}{\Gamma\left(\theta_{1}+\alpha+1\right)}\left(k_{0}+k_{1} R^{m_{1}}+k_{2} R^{m_{2}}\right) \\
& +\left|\frac{1}{1-a \triangle_{1}}\right| \frac{1}{\Gamma(\alpha+1)}\left(k_{0}+k_{1} R^{m_{1}}+k_{2} R^{m_{2}}\right) \\
& +\frac{1}{\Gamma(\alpha+1)}\left(k_{0}+k_{1} R^{m_{1}}+k_{2} R^{m_{2}}\right)=\left|1+\tau_{1}\right| K R^{\mu} \\
& +\left(k_{0}+k_{1} R^{m_{1}}+k_{2} R^{m_{2}}\right)\left[\left|\frac{a}{1-a \triangle_{1}}\right| \frac{1}{\Gamma\left(\theta_{1}+\alpha+1\right)}\right. \\
& \left.+\left|\frac{1}{1-a \triangle_{1}}\right| \frac{1}{\Gamma(\alpha+1)}+\frac{1}{\Gamma(\alpha+1)}\right] \leq\left|1+\tau_{1}\right| K R^{\mu}
\end{aligned}
$$

On the other hand, by Definition 1, we can obtain

$$
{ }^{C} D_{0^{+}}^{\delta} T_{1}(x, y)(t)=\frac{1}{\Gamma(1-\delta)} \int_{0}^{t} \frac{T_{1}(x, y)^{\prime}(s)}{(t-s)^{\delta}} d s .
$$

Repeating a process similar to that of (31), it is easy to see

$$
\begin{aligned}
& \left|T_{1}(x, y)^{\prime}(t)\right| \leq\left|\tau_{1}\right| K R^{\mu}+\left|\frac{a}{1-a \Delta_{1}}\right| \\
& \cdot \frac{1}{\Gamma\left(\alpha+\theta_{1}+1\right)}\left(k_{0}+k_{1} R^{m_{1}}+k_{2} R^{m_{2}}\right) \\
& +\left|\frac{1}{1-a \triangle_{1}}\right| \frac{1}{\Gamma(\alpha+1)}\left(k_{0}+k_{1} R^{m_{1}}+k_{2} R^{m_{2}}\right) \\
& +\frac{1}{\Gamma(\alpha)}\left(k_{0}+k_{1} R^{m_{1}}+k_{2} R^{m_{2}}\right)=\left|\tau_{1}\right| K R^{\mu}+\left(k_{0}\right. \\
& \left.+k_{1} R^{m_{1}}+k_{2} R^{m_{2}}\right)\left[\left|\frac{a}{1-a \triangle_{1}}\right| \frac{1}{\Gamma\left(\alpha+\theta_{1}+1\right)}\right. \\
& \left.+\left|\frac{1}{1-a \triangle_{1}}\right| \frac{1}{\Gamma(\alpha+1)}+\frac{1}{\Gamma(\alpha)}\right] \leq\left|\tau_{1}\right| K R^{\mu} \\
& +C_{1}\left(k_{0}+k_{1} R^{m_{1}}+k_{2} R^{m_{2}}\right) .
\end{aligned}
$$

It follows from (32) and (33) that

$$
\begin{aligned}
& \left|{ }^{C} D_{0^{+}}^{\delta} T_{1}(x, y)(t)\right| \leq \frac{1}{\Gamma(1-\delta)} \int_{0}^{t} \frac{\left|T_{1}(x, y)^{\prime}(s)\right|}{(t-s)^{\delta}} d s \\
& \quad \leq \frac{1}{\Gamma(1-\delta)} \\
& \quad \cdot \int_{0}^{t} \frac{\left|\tau_{1}\right| K R^{\mu}+C_{1}\left(k_{0}+k_{1} R^{m_{1}}+k_{2} R^{m_{2}}\right)}{(t-s)^{\delta}} d s \\
& \quad \leq \frac{1}{\Gamma(2-\delta)}\left[\left|\tau_{1}\right| K R^{\mu}+C_{1}\left(k_{0}+k_{1} R^{m_{1}}+k_{2} R^{m_{2}}\right)\right] .
\end{aligned}
$$

By virtue of (31) and (34), we have

$$
\begin{aligned}
\left\|T_{1}(x, y)\right\|_{X} & \leq B_{1} K R^{\mu}+\left(k_{0}+k_{1} R^{m_{1}}+k_{2} R^{m_{2}}\right) D_{1} \\
& \leq \frac{R}{8}+\frac{R}{8}+\frac{R}{8}+\frac{R}{8}=\frac{R}{2} .
\end{aligned}
$$

Similarly, we can get

$$
\begin{aligned}
\left\|T_{2}(x, y)\right\|_{Y} & \leq B_{2} L R^{\lambda}+\left(l_{0}+l_{1} R^{n_{1}}+l_{2} R^{n_{2}}\right) D_{2} \\
& \leq \frac{R}{8}+\frac{R}{8}+\frac{R}{8}+\frac{R}{8}=\frac{R}{2} .
\end{aligned}
$$

Therefore,

$$
\begin{aligned}
\|T(x, y)\|_{X \times Y} & =\left\|T_{1}(x, y)\right\|_{X}+\left\|T_{2}(x, y)\right\|_{Y} \\
& \leq \frac{R}{2}+\frac{R}{2} \leq R,
\end{aligned}
$$

which means that $T: B_{R} \rightarrow B_{R}$. 
From the continuity of the functions $f, g, h$, and $\varphi$, it is not difficult to see that $T$ is continuous.

Next we show that $T\left(B_{R}\right)$ are equicontinuous. For this sake, let

$$
\begin{aligned}
& M_{1}=\max _{t \in[0,1],|x| \leq R,|y| \leq R}|f(t, x, y)|, \\
& M_{2}=\max _{t \in[0,1],|x| \leq R,|y| \leq R}|g(t, x, y)| .
\end{aligned}
$$

For any $t_{1}, t_{2} \in[0,1]\left(t_{1}<t_{2}\right)$, one has

$$
\begin{aligned}
& \left|T_{1}(x, y)\left(t_{2}\right)-T_{1}(x, y)\left(t_{1}\right)\right| \leq\left|\tau_{1}\right| K R^{\mu}\left|t_{2}-t_{1}\right| \\
& +\left|\frac{a}{1-a \triangle_{1}}\right| \frac{M_{1}}{\Gamma\left(\alpha+\theta_{1}+1\right)}\left|t_{2}-t_{1}\right|+\frac{M_{1}}{\left|1-a \triangle_{1}\right|} \\
& \cdot \frac{1}{\Gamma(\alpha+1)}\left|t_{2}-t_{1}\right| \\
& +\mid \frac{1}{\Gamma(\alpha)} \int_{0}^{t_{2}}\left(t_{2}-s\right)^{\alpha-1} f\left(s, y(s),{ }^{C} D_{0^{+}}^{\gamma} y(s)\right) d s \\
& -\frac{1}{\Gamma(\alpha)} \int_{0}^{t_{1}}\left(t_{1}-s\right)^{\alpha-1} f\left(s, y(s),{ }^{C} D_{0^{+}}^{\gamma} y(s)\right) d s \mid \\
& \quad \leq\left|\tau_{1}\right| K R^{\mu}\left|t_{2}-t_{1}\right|+\left|\frac{a}{1-a \triangle_{1}}\right| \frac{M_{1}}{\Gamma\left(\alpha+\theta_{1}+1\right)} \mid t_{2} \\
& -t_{1}\left|+\frac{M_{1}}{\left|1-a \triangle_{1}\right|} \frac{1}{\Gamma(\alpha+1)}\right| t_{2}-t_{1}\left|+\frac{M_{1}}{\Gamma(\alpha+1)}\right| t_{2}^{\alpha} \\
& -t_{1}^{\alpha} \mid .
\end{aligned}
$$

On the other hand, we have

$$
\begin{gathered}
\left|{ }^{C} D_{0^{+}}^{\delta} T_{1}(x, y)\left(t_{2}\right)-{ }^{C} D_{0^{+}}^{\delta} T_{1}(x, y)\left(t_{1}\right)\right|=\mid \frac{1}{\Gamma(1-\delta)} \\
\cdot \int_{0}^{t_{2}} \frac{T_{1}(x, y)^{\prime}(s)}{\left(t_{2}-s\right)^{\delta}} d s-\frac{1}{\Gamma(1-\delta)} \\
\quad \cdot \int_{0}^{t_{1}} \frac{T_{1}(x, y)^{\prime}(s)}{\left(t_{1}-s\right)^{\delta}} d s \mid \\
\leq \frac{1}{\Gamma(1-\delta)}\left[\int_{0}^{t_{1}} \frac{\left(t_{2}-s\right)^{\delta}-\left(t_{1}-s\right)^{\delta}}{\left(t_{1}-s\right)^{\delta}\left(t_{2}-s\right)^{\delta}} \mid T_{1}(x, y)^{\prime}\right. \\
\left.\cdot(s)\left|d s+\int_{t_{1}}^{t_{2}} \frac{1}{\left(t_{2}-s\right)^{\delta}}\right| T_{1}(x, y)^{\prime}(s) \mid d s\right] .
\end{gathered}
$$

Notice that

$$
\left|T_{1}(x, y)^{\prime}(s)\right| \leq\left|\tau_{1}\right| K R^{\mu}+M_{1} C_{1}
$$

This together with (39)-(40) guarantees that

$$
\begin{aligned}
& \left|{ }^{C} D_{0^{+}}^{\delta} T_{1}(x, y)\left(t_{2}\right)-{ }^{C} D_{0^{+}}^{\delta} T_{1}(x, y)\left(t_{1}\right)\right| \\
& \quad \leq \frac{1}{\Gamma(1-\delta)}\left(\left|\tau_{1}\right| K R^{\mu}+M_{1} C_{1}\right) \\
& \quad \cdot\left[\int_{0}^{t_{1}} \frac{\left(t_{2}-s\right)^{\delta}-\left(t_{1}-s\right)^{\delta}}{\left(t_{1}-s\right)^{\delta}\left(t_{2}-s\right)^{\delta}} d s+\int_{t_{1}}^{t_{2}} \frac{1}{\left(t_{2}-s\right)^{\delta}} d s\right] \\
& \quad \leq \frac{1}{\Gamma(2-\delta)}\left(\left|\tau_{1}\right| K R^{\mu}+M_{1} C_{1}\right) \\
& \quad \cdot\left[t_{2}^{1-\delta}-t_{1}^{1-\delta}+2\left(t_{2}-t_{1}\right)^{1-\delta}\right] .
\end{aligned}
$$

Similarly, we can get

$$
\begin{aligned}
& \left|T_{2}(x, y)\left(t_{2}\right)-T_{2}(x, y)\left(t_{1}\right)\right| \leq\left|\tau_{2}\right| L R^{\lambda}\left|t_{2}-t_{1}\right| \\
& \quad+\left|\frac{b}{1-b \triangle_{2}}\right| \frac{M_{2}}{\Gamma\left(\beta+\theta_{2}+1\right)}\left|t_{2}-t_{1}\right|+\frac{M_{2}}{\left|1-b \triangle_{2}\right|} \\
& \quad \cdot \frac{1}{\Gamma(\beta+1)}\left|t_{2}-t_{1}\right|+\frac{M_{2}}{\Gamma(\beta+1)}\left|t_{2}^{\beta}-t_{1}^{\beta}\right| . \\
& \left|{ }^{C} D_{0^{+}}^{\gamma} T_{2}(x, y)\left(t_{2}\right)-{ }^{C} D_{0^{+}}^{\gamma} T_{2}(x, y)\left(t_{1}\right)\right| \\
& \quad \leq \frac{1}{\Gamma(2-\gamma)}\left(\left|\tau_{2}\right| L R^{\lambda}+M_{2} C_{2}\right) \\
& \quad \cdot\left[t_{2}^{1-\gamma}-t_{1}^{1-\gamma}+2\left(t_{2}-t_{1}\right)^{1-\gamma}\right] .
\end{aligned}
$$

This together with (39), (42), and (43) implies that $T\left(B_{R}\right)$ are equicontinuous. By virtue of the Arzela-Ascoli theorem, we can infer that the operator $T$ is completely continuous. Hence, applying Schauder fixed theorem, FDE (4) has at least one solution $(x, y)$ in $B_{R}$.

Theorem 11. Assume that $\left(H_{2}\right)-\left(H_{4}\right)$ hold. Then FDE (4) has at least one solution.

Proof. Firstly, we show that $T$ is completely continuous operator. From the continuity of the functions $f, g, h$, and $\varphi$, it follows that the operator $T$ is continuous. Let $B_{Q}=\{(x, y) \in$ $\left.X \times Y \mid\|(x, y)\|_{X \times Y} \leq Q\right\}$, where $Q$ is described as in $\left(H_{4}\right)$; we now prove that $T\left(B_{\mathrm{Q}}\right)$ is relatively compact.

For any $(x, y) \in B_{Q}$, by similar computation as (31) and (34), we obtain that

$$
\begin{aligned}
& \left|T_{1}(x, y)(t)\right| \leq\left|1+\tau_{1}\right| K Q^{\mu}+A_{1}\left(a_{1}+b_{1} \phi_{1}(Q)\right. \\
& \left.\quad+c_{1} \psi_{1}(Q)\right) \\
& \left|{ }^{C} D_{0^{+}}^{\delta} T_{1}(x, y)(t)\right| \leq \frac{1}{\Gamma(2-\delta)}\left[\left|\tau_{1}\right| K Q^{\mu}\right. \\
& \left.\quad+C_{1}\left(a_{1}+b_{1} \phi_{1}(Q)+c_{1} \psi_{1}(Q)\right)\right]
\end{aligned}
$$


which imply

$$
\begin{aligned}
\left\|T_{1}(x, y)(t)\right\|_{X} \leq & \left(\left|1+\tau_{1}\right|+\frac{\left|\tau_{1}\right|}{\Gamma(2-\delta)}\right) K Q^{\mu} \\
& +D_{1}\left(a_{1}+b_{1} \phi_{1}(Q)+c_{1} \psi_{1}(Q)\right) .
\end{aligned}
$$

Similarly, we have

$$
\begin{aligned}
\left\|T_{2}(x, y)(t)\right\|_{Y} \leq & \left(\left|1+\tau_{2}\right|+\frac{\left|\tau_{2}\right|}{\Gamma(2-\gamma)}\right) L Q^{\lambda} \\
& +D_{2}\left(a_{2}+b_{2} \phi_{2}(Q)+c_{2} \psi_{2}(Q)\right) .
\end{aligned}
$$

Combining (45) and (46), one can get

$$
\begin{aligned}
\|T(x, y)\|_{X \times Y} \leq & \left(\left|1+\tau_{1}\right|+\frac{\left|\tau_{1}\right|}{\Gamma(2-\delta)}\right) K Q^{\mu} \\
& +D_{1}\left(a_{1}+b_{1} \phi_{1}(Q)+c_{1} \psi_{1}(Q)\right) \\
& +\left(\left|1+\tau_{2}\right|+\frac{\left|\tau_{2}\right|}{\Gamma(2-\gamma)}\right) L Q^{\lambda} \\
& +D_{2}\left(a_{2}+b_{2} \phi_{2}(Q)+c_{2} \psi_{2}(Q)\right) \\
= & B_{1} K Q^{\mu}+D_{1} P_{1}+B_{2} L Q^{\lambda}+D_{2} P_{2} .
\end{aligned}
$$

By (47) and $\left(H_{4}\right)$ we know $T\left(B_{Q}\right) \subset B_{Q}$. Using similar computation as in (39), (40), (42), and (43), we can obtain $T\left(B_{\mathrm{Q}}\right)$ are equicontinuous. Thus, $T$ is completely continuous by Arzela-Ascoli theorem.

We now claim that $T$ has at least one solution in $X \times Y$.

Suppose there exists $(x, y) \in \partial B_{\mathrm{Q}}$ such that $(x, y)=$ $\lambda T(x, y)$ for some $\lambda \in(0,1)$. This together with (44) implies that

$$
\begin{aligned}
|x(t)| & =\lambda\left|T_{1}(x, y)(t)\right| \leq\left|T_{1}(x, y)(t)\right|, \\
\left|{ }^{C} D_{0^{+}}^{\delta} x(t)\right| & =\lambda\left|{ }^{C} D_{0^{+}}^{\delta} T_{1}(x, y)(t)\right| \\
& \leq\left|{ }^{C} D_{0^{+}}^{\delta} T_{1}(x, y)(t)\right| .
\end{aligned}
$$

By virtue of (44)-(47), it is easy to see

$$
\|(x, y)\|_{X \times Y} \leq B_{1} K Q^{\mu}+D_{1} P_{1}+B_{2} L Q^{\lambda}+D_{2} P_{2}<Q .
$$

This is a contradiction with $(x, y) \in \partial B_{Q}$. Therefore, it follows from Lemma 9 that the operator $T$ has a fixed point $(x, y) \in$ $\bar{B}_{\mathrm{Q}}$. Thus FDE (4) has at least one solution in $X \times Y$.

Remark 12. Comparing $\left(H_{1}\right)$ with $\left(H_{3}\right)$, we know that condition $\left(H_{3}\right)$ is more extensive than $\left(H_{1}\right)$. However, the assumptions of Theorem 10 are easier to verify than that of Theorem 11.

\section{Examples}

Example 1. Consider the following coupled system:

$$
\begin{aligned}
{ }^{C} D_{0^{+}}^{5 / 4} x(t)= & \frac{1}{\sqrt{25+t^{2}}} \cos t+\frac{1}{2}(y(t))^{1 / 5} \\
& +\frac{1}{4(1+t)}\left({ }^{C} D_{0^{+}}^{1 / 4} y(t)\right)^{1 / 3}, \\
{ }^{C} D_{0^{+}}^{3 / 2} y(t)= & \frac{e^{-t}}{3+t^{2}}+\frac{1}{4}(x(t))^{1 / 7} t \in[0,1], \\
& +\frac{1}{4(2+t)} \sin \left({ }^{C} D_{0^{+}}^{1 / 2} x(t)\right)^{1 / 5}, \\
x(0)= & \frac{1}{4}\left(\int_{0}^{1} x(t) d t\right)^{1 / 7}, \\
x(1)= & 3 I_{0^{+}}^{1 / 4} x\left(\frac{1}{3}\right), \\
y(1)= & 2 I_{0^{+}}^{1 / 2} y\left(\frac{1}{2}\right), \\
y(0)= & \frac{1}{2}\left(\int_{0}^{1} y(t) d t\right)^{1 / 5},
\end{aligned}
$$

where $\alpha=5 / 4, \beta=3 / 2, \gamma=1 / 4, \delta=1 / 2, a=3, b=2$, $\theta_{1}=1 / 4, \theta_{2}=1 / 2, \eta=1 / 3, \xi=1 / 2$.

(50) can be regarded as the form of (4), where

$$
\begin{aligned}
f(t, u, v)= & \frac{1}{\sqrt{25+t^{2}}} \cos t+\frac{1}{2}(u(t))^{1 / 5} \\
& +\frac{1}{4(1+t)}(v(t))^{1 / 3} \\
g(t, u, v)= & \frac{e^{-t}}{3+t^{2}}+\frac{1}{4}(u(t))^{1 / 7} \\
& +\frac{1}{4(2+t)} \sin (v(t))^{1 / 5}, \\
h(x)= & \frac{1}{4}\left(\int_{0}^{1} x(t) d t\right)^{1 / 7}, \\
\varphi(y)= & \frac{1}{2}\left(\int_{0}^{1} y(t) d t\right)^{1 / 5} .
\end{aligned}
$$

It is not difficult to see

$$
\begin{aligned}
& |f(t, u, v)| \leq \frac{1}{5}+\frac{1}{2}(|u|)^{1 / 5}+\frac{1}{4}(|v|)^{1 / 3}, \\
& |g(t, u, v)| \leq \frac{1}{3}+\frac{1}{4}(|u|)^{1 / 7}+\frac{1}{8}(|v|)^{1 / 5},
\end{aligned}
$$




$$
\begin{aligned}
& |h(x)| \leq \frac{1}{4}\|x\|_{c}^{1 / 7}, \\
& |\varphi(y)| \leq \frac{1}{2}\|y\|_{c}^{1 / 5} .
\end{aligned}
$$

Choose $k_{0}=1 / 5, k_{1}=1 / 2, k_{2}=1 / 4, l_{0}=1 / 3, l_{1}=1 / 4$, $l_{2}=1 / 8, m_{1}=1 / 5, m_{2}=1 / 3, n_{1}=1 / 7, n_{2}=1 / 5, K=1 / 4$, $L=1 / 2, \mu=1 / 7, \lambda=1 / 5$. Obviously, $h(0)=\varphi(0)=0$. Therefore, $\left(H_{1}\right)$ and $\left(H_{2}\right)$ are satisfied. Immediately, we can conclude that FDE (4) has at least one solution by Theorem 10.

Example 2. Consider the following problem:

$$
\begin{aligned}
{ }^{C} D_{0^{+}}^{3 / 2} x(t)= & \frac{(1-t)^{2}}{3000}+\frac{y^{2}(t)}{7000(1+|y|)} \\
& +\frac{t}{5000}\left({ }^{C} D_{0^{+}}^{1 / 2} y(t)\right)^{2}, \quad t \in[0,1], \\
{ }^{C} D_{0^{+}}^{5 / 4} y(t)= & \frac{(1+t)^{2}}{4000}+\frac{(1-t) x^{4}(t)}{4000\left(1+x^{2}\right)} \\
& +\frac{t^{2}}{1000}\left({ }^{C} D_{0^{+}}^{1 / 2} x(t)\right)^{3}, \quad t \in[0,1], \\
x(0)= & \left.\frac{1}{200}\left(\max _{t \in[0,1]}|x(t)|\right)\right)^{1 / 3}, \\
x(1)= & 4 I_{0^{+}}^{1 / 4} x\left(\frac{1}{4}\right), \\
y(0)= & \left.\frac{1}{400}\left(\max _{t \in[0,1]}|y(t)|\right)\right)^{1 / 2}, \\
y(1)= & 2 I_{0^{+}}^{1 / 2} y\left(\frac{1}{2}\right),
\end{aligned}
$$

where $\alpha=3 / 2, \beta=5 / 4, \gamma=1 / 2, \delta=1 / 2, a=4, b=2$, $\theta_{1}=1 / 4, \theta_{2}=1 / 2, \eta=1 / 4, \xi=1 / 2$.

(53) can be regarded as the form of (4), where

$$
\begin{aligned}
f(t, u, v) & =\frac{(1-t)^{2}}{3000}+\frac{u^{2}}{7000(1+|u|)}+\frac{t}{5000} v^{2}, \\
g(t, u, v) & =\frac{(1+t)^{2}}{4000}+\frac{(1-t) u^{4}}{4000\left(1+u^{2}\right)}+\frac{t^{2}}{1000} v^{3}, \\
h(x) & =\frac{1}{200}\left(\max _{t \in[0,1]}|x(t)|\right)^{1 / 3}, \\
\varphi(y) & =\frac{1}{400}\left(\max _{t \in[0,1]}|y(t)|\right)^{1 / 2} .
\end{aligned}
$$

It is not difficult to see

$$
\begin{aligned}
& |f(t, u, v)| \leq \frac{1}{3000}+\frac{|u|^{2}}{7000}+\frac{|v|^{2}}{5000}, \\
& |g(t, u, v)| \leq \frac{1}{1000}+\frac{1}{4000}|u|^{2}+\frac{1}{1000}|v|^{3},
\end{aligned}
$$

$$
\begin{aligned}
& |h(x)| \leq \frac{1}{200}\|x\|_{c}^{1 / 3}, \\
& |\varphi(y)| \leq \frac{1}{400}\|y\|_{c}^{1 / 2} .
\end{aligned}
$$

Choose $a_{1}=1 / 3000, b_{1}=1 / 7000, c_{1}=1 / 5000, \phi_{1}(|u|)=$ $|u|^{2}, \psi_{1}(|v|)=|v|^{2}, a_{2}=1 / 1000, b_{2}=1 / 4000, c_{2}=1 / 1000$, $\phi_{2}(|u|)=|u|^{2}, \psi_{2}(|v|)=|v|^{3}, K=1 / 200, L=1 / 400, \mu=1 / 3$, $\lambda=1 / 2$. Now we need to verify $\left(H_{4}\right)$. By direct calculation, it is easy to see

$$
\begin{aligned}
& \triangle_{1}=0.0781, \\
& \sigma_{1}=0.7801, \\
& \tau_{1}=3.0838, \\
& \triangle_{2}=0.2660, \\
& \sigma_{2}=0.1995, \\
& \tau_{2}=-1.2842, \\
& A_{1}=11.2022, \\
& B_{1}=4.9537, \\
& C_{1}=10.7321, \\
& D_{1}=14.2296, \\
& P_{1}=0.0051, \\
& A_{2}=5.9608, \\
& B_{2}=0.6447, \\
& C_{2}=5.9846, \\
& D_{2}=7.6490, \\
& P_{2}=0.0690,
\end{aligned}
$$

Thus, we can get $B_{1} K Q^{\mu}+D_{1} P_{1}+B_{2} L Q^{\lambda}+D_{2} P_{2}=0.0394+$ $0.0726+0.0032+0.5278=0.7582<4$ for $Q=4$, which means that $\left(\mathrm{H}_{4}\right)$ holds. Consequently, $\mathrm{FDE}(4)$ has at least one solution by Theorem 11 .

\section{Conflicts of Interest}

The authors declare that there are no conflicts of interest regarding the publication of this paper.

\section{Acknowledgments}

This project is supported by NNSF of China (11671237) and NSF of Shandong Province (ZR2013AM005).

\section{References}

[1] I. Podlubny, Fractional Differential Equations, Mathematics in Science and Engineering, vol. 198, Academic Press, San Diego, Calif, USA, 1999. 
[2] J. Sabatier, O. P. Agrawal, and J. A. T. Machado, Advances in Fractional Calculus: Theoretical Developments and Applications in Physics and Engineering, Springer-Verlag, Berlin, Germany, 2007.

[3] R. Hilfer, Applications of Fractional Calculus in Physics, World Scientific, Singapore, 2000.

[4] R. L. Magin, "Fractional calculus in bioengineering," Critical Reviews in Biomedical Engineering, vol. 32, no. 1, pp. 1-377, 2004.

[5] K. B. Oldham, "Fractional differential equations in electrochemistry," Advances in Engineering Software, vol. 41, no. 1, pp. 9-12, 2010.

[6] B. Liu and Y. Liu, "Positive solutions of a two-point boundary value problem for singular fractional differential equations in banach space," Journal of Function Spaces and Applications, vol. 2013, Article ID 585639, 9 pages, 2013.

[7] Y. Cui, "Uniqueness of solution for boundary value problems for fractional differential equations," Applied Mathematics Letters, vol. 51, pp. 48-54, 2016.

[8] J. Jiang, L. Liu, and Y. Wu, "Positive solutions to singular fractional differential system with coupled boundary conditions," Communications in Nonlinear Science and Numerical Simulation, vol. 18, no. 11, pp. 3061-3074, 2013.

[9] H. Li, X. Kong, and C. Yu, "Existence of three non-negative solutions for a three-point boundary-value problem of nonlinear fractional differential equations," Electronic Journal of Differential Equations, vol. 2012, no. 2, pp. 596-602, 2012.

[10] S. Zhang, "Positive solutions for boundary-value problems of nonlinear fractional differential equations," Electronic Journal of Differential Equations, vol. 2006, no. 36, pp. 1-12, 2006.

[11] R. P. Agarwal, D. O’Regan, and S. Stanek, "Positive solutions for Dirichlet problems of singular nonlinear fractional differential equations," Journal of Mathematical Analysis and Applications, vol. 371, no. 1, pp. 57-68, 2010.

[12] A. Guezane-Lakoud and R. Khaldi, "Solvability of a fractional boundary value problem with fractional integral condition," Nonlinear Analysis. Theory, Methods \& Applications, vol. 75, no. 4, pp. 2692-2700, 2012.

[13] X. Su, "Boundary value problem for a coupled system of nonlinear fractional differential equations," Applied Mathematics Letters, vol. 22, no. 1, pp. 64-69, 2009.

[14] X. Zhang, Y. Wu, and L. Caccetta, "Nonlocal fractional order differential equations with changing-sign singular perturbation," Applied Mathematical Modelling. Simulation and Computation for Engineering and Environmental Systems, vol. 39, no. 21, pp. 6543-6552, 2015.

[15] Z. Han, H. Lu, and C. Zhang, "Positive solutions for eigenvalue problems of fractional differential equation with generalized $p$ Laplacian," Applied Mathematics and Computation, vol. 257, pp. 526-536, 2015.

[16] S. Sun, Y. Zhao, Z. Han, and M. Xu, "Uniqueness of positive solutions for boundary value problems of singular fractional differential equations," Inverse Problems in Science and Engineering, vol. 20, no. 3, pp. 299-309, 2012.

[17] Y. Cui and Y. Zou, "An existence and uniqueness theorem for a second order nonlinear system with coupled integral boundary value conditions," Applied Mathematics and Computation, vol. 256, pp. 438-444, 2015.

[18] Y. Cui and Y. Zou, "Existence of solutions for second-order integral boundary value problems," Lithuanian Association of Nonlinear Analysts (LANA). Nonlinear Analysis. Modelling and Control, vol. 21, no. 6, pp. 828-838, 2016.
[19] D. Zhao and Y. Liu, "Positive solutions for a class of fractional differential coupled system with integral boundary value conditions," Journal of Nonlinear Science and its Applications. JNSA, vol. 9, no. 5, pp. 2922-2942, 2016.

[20] Y. Li, Y. Sang, and H. Zhang, "Solvability of a coupled system of nonlinear fractional differential equations with fractional integral conditions," Journal of Applied Mathematics and Computing, vol. 50, no. 1-2, pp. 73-91, 2016.

[21] B. Ahmad, S. K. Ntouyas, and A. Alsaedi, "On a coupled system of fractional differential equations with coupled nonlocal and integral boundary conditions," Chaos, Solitons \& Fractals, vol. 83, pp. 234-241, 2016.

[22] A. A. Kilbas, H. M. Srivastava, and J. J. Trujillo, Theory and Applications of Fractional Differential Equations, in:NorthHolland Mathematics studies, Elsevier Science, Amsterdaam, Netherlands, 2006.

[23] K. Deimling, Nonlinear Functional Analysis, Springer, Berlin, Germany, 1985. 


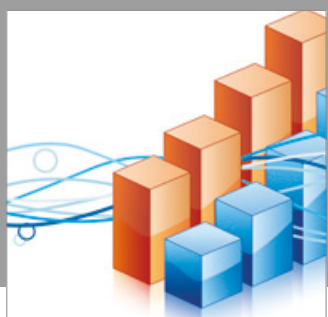

Advances in

Operations Research

vatersals

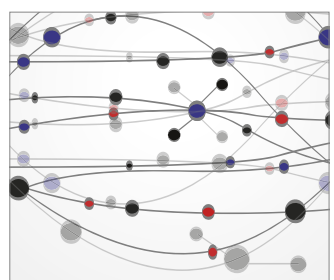

\section{The Scientific} World Journal
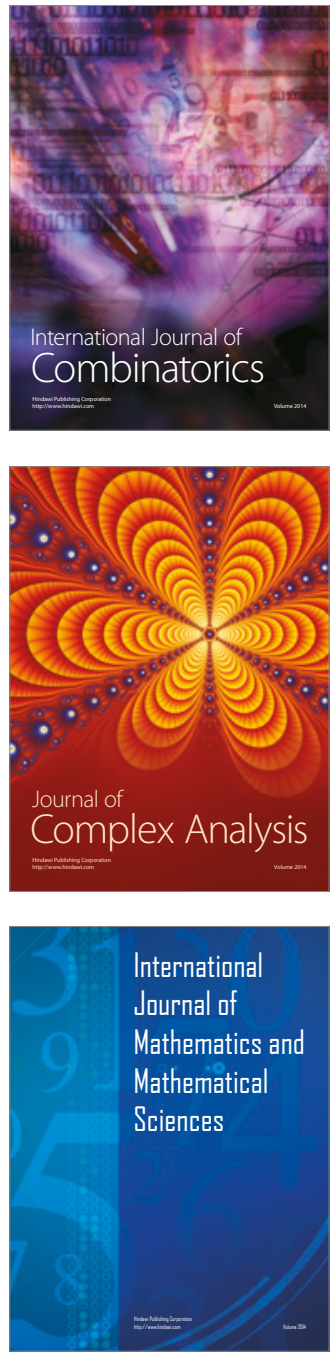
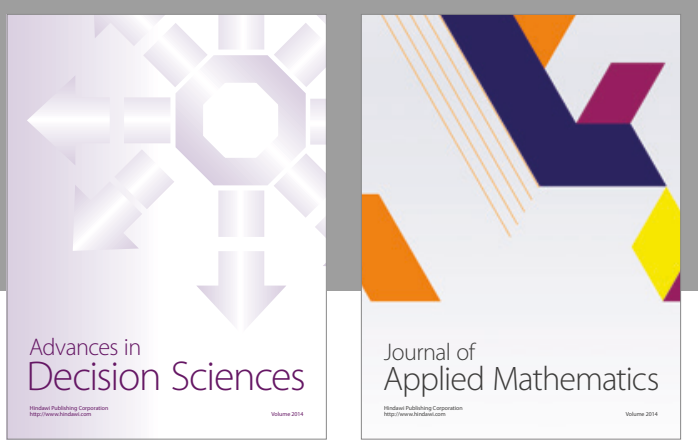

Algebra

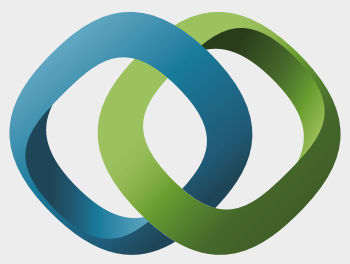

\section{Hindawi}

Submit your manuscripts at

https://www.hindawi.com
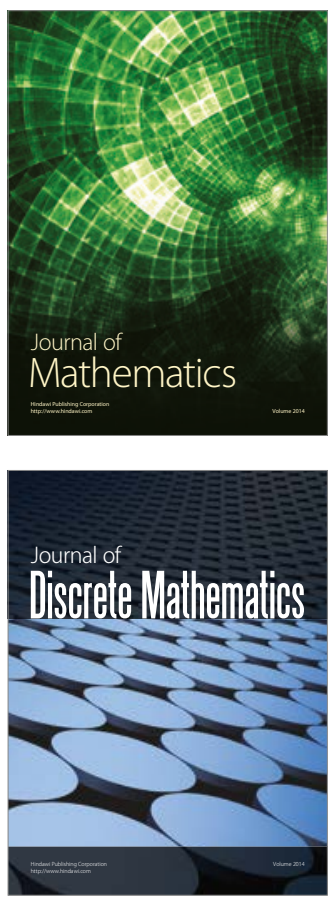

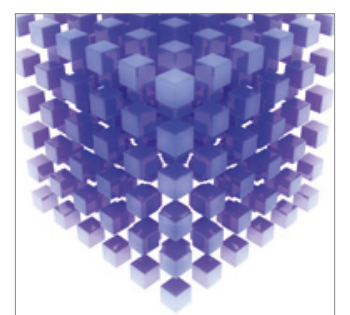

Mathematical Problems in Engineering
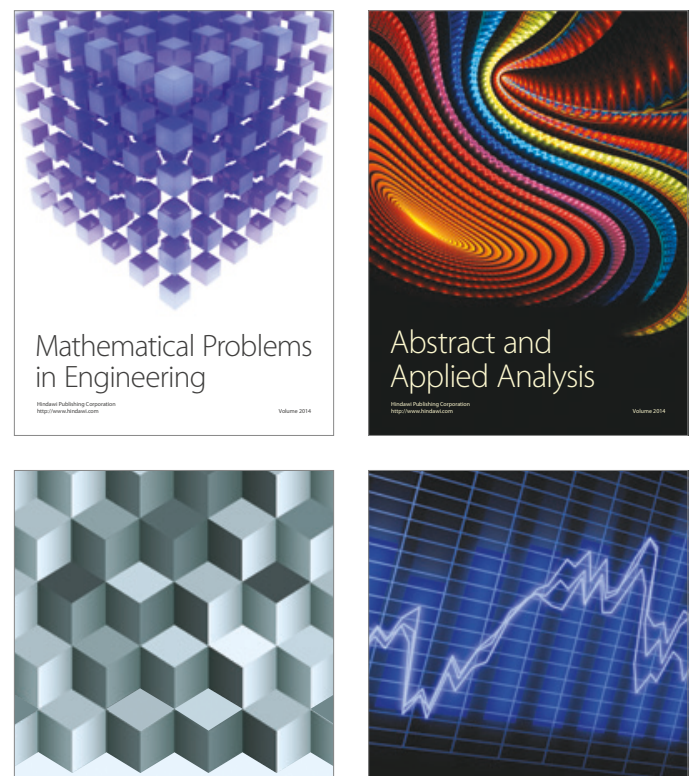

Journal of

Function Spaces

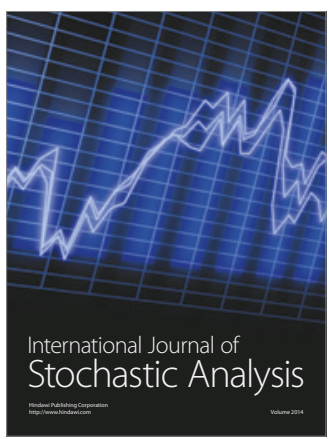

Probability and Statistics
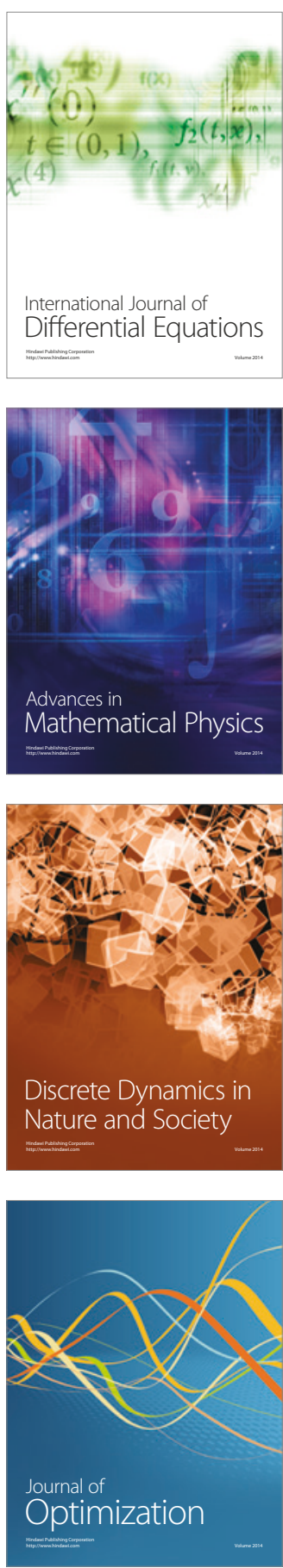